\title{
474662 - HEMODYNAMIC RESPONSE AND AIRWAY MORBIDITY FOLLOWING TRACHEAL INTUBATION: A COMPARISON BETWEEN DIRECT LARYNGOSCOPY, VIDEO LARYNGOSCOPY AND LIGHTWAND TECHNIQUES
}

\author{
Naveed Siddiqui, M.D ${ }^{1}$, Rita Katznelson, M.D. ${ }^{2}$, Zeev Friedman, M.D. ${ }^{1}$ \\ 1. Anesthesia and Pain Management, Mount Sinai Hospital, University of Toronto, \\ Toronto, ON, Canada \\ 2. Department of Anesthesia, Toronto General Hospital,University of Toronto, \\ Toronto, ON, Canada
}

Introduction: Airway manipulation during direct laryngoscopy (DL) and intubation can produce a marked physiological response. These changes can be detrimental in scenarios such as ischemic heart disease, aortic dissection or intracranial hemorrhage $(1,2)$. Minimizing oropharyngo-laryngeal stimulation or avoiding laryngoscopy may attenuate the hemodynamic response. In this prospective randomized control trial, we compared the hemodynamic response following tracheal intubation, using three different techniques of intubation. We also examined post-operative airway morbidities, as our secondary outcome.

Methods: Local REB approval was obtained for this study. Sixty, adult ASA I-II patients, scheduled for elective surgery requiring general anesthesia with oro-tracheal intubation were randomly allocated into one of 3 groups $(n=20)$. DL group - endotracheal intubation was achieved with a Macintosh laryngoscope. GS group- intubation using a Glidescope. TL group- trachlight intubation. Anesthesia technique was standardized, and intubation was performed by a single experienced anesthesiologist. The hemodynamic variables, mean arterial pressure (MAP) and heart rate (HR) were measured noninvasively before induction (baseline values) immediately after induction (post induction values), at intubation, and for 5 minutes after intubation at one-minute intervals. We also recorded the number of attempts and total time for intubation using the three techniques. The severity of post-operative airway morbidity upto 48 hours following surgery was assessed using a questionnaire and an analog visual scale (VAS).

Results: There was no significant difference in MAP and HR when compared between the groups (graph 1-2). DL intubation times were significantly lower than those in the GS and TL groups (both $\mathrm{p}<0.0001$ ). Sore throat and VAS assessed in the PACU were significantly higher in the TL group than in the DL group $(\mathrm{p}=0.0033)$.

Discussion: No benefit was achieved by using any of the 3 intubation techniques for attenuation of hemodynamic changes. There was a higher incidence of sore throat associated with trachlight intubation than with DL and GS.

References: (1) Br J Anaesth 1970; 42: 618-24. (2) Anesthesiology 1977; 47: 524-5. 

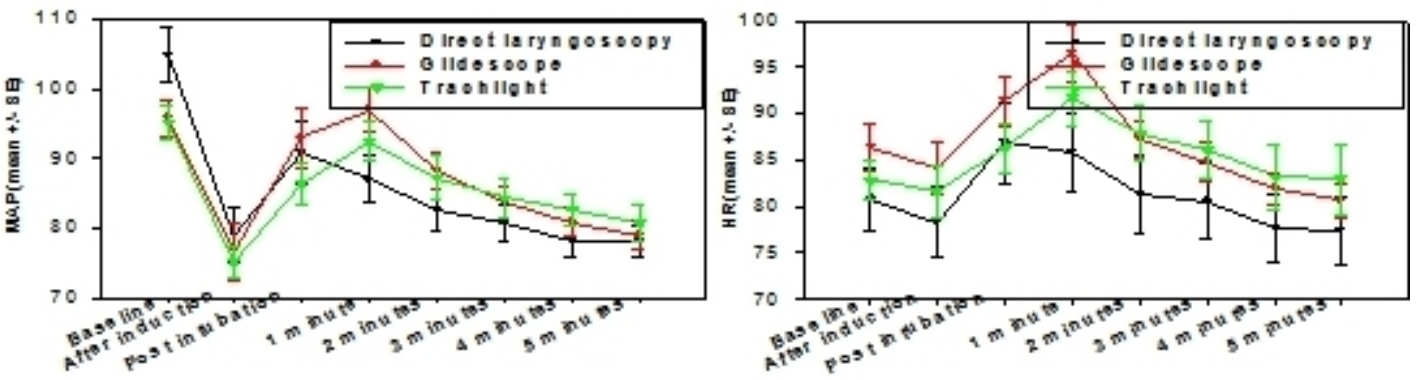

Graph 1-2 